\title{
Obesity is Not Associated with Perioperative Adverse Events in Patients Undergoing Complex Revision Surgery for the Thoracolumbar Spine
}

Takashi Hirase ( $\nabla$ thirase@houstonmethodist.org )

Houston Methodist Hospital

Jeremiah F. Ling

Texas A\&M University Health Science Center College of Medicine

Varan Haghshenas

Houston Methodist Hospital

Richard Fuld 3rd

Houston Methodist Hospital

David Dong

Houston Methodist Hospital

Darrell S. Hanson

Houston Methodist Hospital

\section{B. Christoph Meyer}

Houston Methodist Hospital

Rex A.W. Marco

Houston Methodist Hospital

\section{Research Article}

Keywords: obesity, revision thoracolumbar spine surgery, sarcopenia, complex, predictor.

Posted Date: September 1st, 2021

DOI: https://doi.org/10.21203/rs.3.rs-789945/v1

License: (1) (1) This work is licensed under a Creative Commons Attribution 4.0 International License. Read Full License 


\section{Abstract}

Study Design: Retrospective cohort study

Objective: This study sought to determine the relationship between obesity and perioperative adverse events (AEs) with patients undergoing complex revision thoracolumbar spine surgery while controlling for psoas muscle index (PMI) as a confounding variable.

Methods: Between May 2016 and February 2020, a retrospective analysis of individuals undergoing complex revision surgery of the thoracolumbar spine was performed at a single institution. Obesity was defined as $\mathrm{BMI} \geq 30.0 \mathrm{~kg} / \mathrm{m}^{2}$. PMl $<500 \mathrm{~mm}^{2} / \mathrm{m}^{2}$ for males and $<412 \mathrm{~mm}^{2} / \mathrm{m}^{2}$ for females were used to define sarcopenia. A Spine Surgical Invasiveness Index (SSII) $>10$ was used to define complex revision surgery. A multivariable logistic regression model was used to ascertain the effects of sarcopenia, obesity, age, and gender on the likelihood of the occurrence of any AE.

Results: The study included 114 patients. 54 patients were in the obese cohort and 60 patients in the nonobese cohort. There was not a significant difference in perioperative outcomes of both the obese and non-obese patients. Multivariable analysis demonstrated that sarcopenic individuals had a significantly higher likelihood for an AE than non-sarcopenic individuals (OR: 7.53, 95\% Cl: 3.05-18.60). Obesity did not have a significant effect in predicting AEs.

Conclusions: Obesity is not associated with perioperative AEs, 30-day readmission rates, 30-day reoperation rates, rate of discharge to a facility, or post-operative length of stay (LOS) among patients undergoing complex revision thoracolumbar spine surgery.

\section{Level of Evidence: III}

\section{Introduction}

Currently, over 100 million adults living in the United States are considered obese and overweight. ${ }^{1}$ The $\mathrm{NIH}$ utilizes body mass index (BMI) as a parameter of categorizing weight status, calculating $\mathrm{BMI}$ using body weight in kilograms dived by height in meters squared. ${ }^{2}$ Obesity is a chronic disease recognized clinically as having a BMI $>30$ in adults and has association with other comorbidities such as Type 2 Diabetes, cardiovascular disease and dyslipidemia. ${ }^{3}$ The impact of obesity on spinal surgeries remains unclear, and complications related to obesity have been reported in some studies. ${ }^{4,5}$ Studies by Shamji et al. and Manson et al. found that obesity is associated with increased resources and perioperative transfusion requirements after elective thoracolumbar spine surgery; however, they reported no other associations between obesity and increased adverse events (AEs) ${ }^{6,7}$ Another study by Yadla et al. investigating outcomes after primary elective thoracolumbar spine surgery found no associates between $\mathrm{BMI}$ and increased AEs. ${ }^{8}$ Furthermore, a more recent study by Varshneya et al. reported that although obesity may be associated with overall health burdens outside of the perioperative environment, there is 
no significant relationship attributed between obesity and AEs after primary thoracolumbar deformity surgery. ${ }^{9}$

Revision thoracolumbar spine surgeries have been associated with significantly higher perioperative AEs compared with primary surgeries. ${ }^{10-12}$ An increase in surgical complexity has also been associated with higher perioperative AEs in spine surgery. ${ }^{13}$ Therefore, identifying specific prognostic indicators for complex revision thoracolumbar spine surgery is important in order to stratify pre-operative risk for these patients. A recent study by Hirase et al. demonstrated that sarcopenia, defined by psoas muscle index (PMI) below $500 \mathrm{~mm}^{2} / \mathrm{m}^{2}$ for males and $412 \mathrm{~mm} / 2 \mathrm{~m}^{2}$ for females, is predictive of increased perioperative AEs among patients undergoing complex revision thoracolumbar spine surgery. ${ }^{14}$ However, this study reported a significantly lower BMl among sarcopenic patients, indicating the presence of a possible confounding factor for predicting perioperative AEs within this patient cohort. Our understanding is that there are no previous studies that evaluate the effect of obesity status on patients undergoing complex revision thoracolumbar spine surgery. Thus, the purpose of this study was to elucidate the relationship between obesity and perioperative $A E s$ among patients undergoing complex revision thoracolumbar spine surgery while controlling for PMI as a confounding variable.

\section{Materials And Methods}

The same patient cohort and similar methodologies were utilized from our previous publication by Hirase et al. ${ }^{14}$ The local institutional review board approved the study procedure on April 13, 2020. Due to the retrospective observational nature of this study, the informed consent was waived.

\section{Study Population}

Between May 2016 and February 2020, a retrospective analysis was performed at a single institution of patients receiving complex revision thoracolumbar spine surgery by three board certified fellowshiptrained orthopaedic spine surgeons. The same inclusion and exclusion criteria for the same cohort of patients from our previous publication by Hirase et al. was applied for this study. ${ }^{14}$ Any patients age 18 or above undergoing complex revision thoracolumbar spine surgery were included. Any history of prior surgical intervention of the same vertebral level was defined as revision surgery. A Spine Surgical Invasiveness Index (SSII) > 10 was used to define complex surgery. ${ }^{18}$ Patients lacking a pre-operative CT or MRI of the lumbar spine obtained at our facility within six months of surgery, poor image quality, preoperative MRI or CT acquired at any outside facilities, clinical evidence of L1 or L2 nerve root compression, a history of previous surgical treatment to or through the psoas muscle, and coronal deformity greater than 20 degrees were excluded as mentioned in our previous study. ${ }^{14}$

\section{Data Collection}


Electronic medical records were utilized retrospectively to obtain demographic data including age, gender, BMI, American Anesthesiologists' Society (ASA) class, comorbidities, indication for revision operation, baseline ambulation status, and presence of neurologic deficits. $B M I \geq 30.0 \mathrm{~kg} / \mathrm{m}^{2}$ was used to define obesity. Intraoperative data was acquired which included estimated blood loss (EBL) and operative time defined as time of incision to post-operative dressing placement. SSII, a verified method of evaluation and comparison regarding spine surgery complexity was used, with a range of $0-48$, which accounts for surgical approaches and the amount of decompressed, fused, and instrumented vertebral levels. ${ }^{18}$ In regard to the included surgical population with revision surgeries, only the additional levels of fusion, instrumentation, or decompression were accounted for the SSII calculation as previously stated. ${ }^{14}$ Each patients' primary surgery SSII score was also obtained.

\section{Assessment Of Sarcopenia}

PMI was used to analyze sarcopenia, which was calculated by measuring the total cross-sectional area (CSA) of the bilateral psoas muscles at the L3 vertebral body using the pre-operative T1 weighted MRI or CT normalized to body height ${ }^{2}\left(\mathrm{~mm}^{2} / \mathrm{m}^{2}\right)$. The total CSA was measured using OsiriX DICOM Viewer software (Version 11.0, Bernex, Switzerland) by manual outlining the bilateral psoas muscles at the first axial cut, done in the craniocaudal direction where both transverse processes are visible at the L3 level. 14,15 All of the images were obtained at a single institution with the same scanning protocols ensuring identical scanning thickness among all images analyzed. Three separate reviewers performed all measurements to improve interobserver reliability. Each measurement was acquired three times by all reviewers to improve intraobserver reliability. Intraclass correlation coefficient (ICC) was used to assess interobserver and intraobserver reliabilities, where an ICC above 0.90 signifies excellent agreement, between 0.75 and 0.90 signifies good agreement, between 0.5 and 0.75 signifies moderate agreement, and below 0.5 signifies poor agreement. ${ }^{16}$ Each of the mean values obtained by the three reviewers was divided by the square of patient height to calculate the PMI as previously described. ${ }^{14}$ To minimize the risk of bias, all reviewers were blinded to their respective measurements as well as to patient demographics and outcomes. Sarcopenia was defined as $\mathrm{PMI}<500 \mathrm{~mm} / \mathrm{m}^{2}$ for males and $<412$ $\mathrm{mm}^{2} / \mathrm{m}^{2}$ for females as previously defined. ${ }^{14}$

\section{Outcome Measures}

Retrospective analysis of electronic medical records were used to review all perioperative outcomes. The primary outcome measures were perioperative AEs which included post-operative anemia that required transfusion, cardiac complication (cardiac arrest and myocardial infarction), sepsis, wound complication (wound dehiscence and deep wound infection), acute kidney injury (AKI), delirium, intra-operative dural tear, pneumonia, urinary tract infection (UTI), urinary retention, epidural hematoma, and deep vein thrombosis (DVT). The secondary outcome measures used were 30-day readmission rates, 30-day reoperation rates, in-hospital mortality rates, discharge disposition (home vs facility) and post-operative 
hospital length of stay (LOS). The number of days from surgery (or the last surgery if staged procedure) to discharge to either home or facility was used to defined postoperative LOS.

\section{Statistical Analysis}

SPSS statistical software (Version 25.0; SPSS, Inc, Chicago, IL) was utilized to perform data analysis. The Chi-Square or Fisher's exact test and continuous data was used to analyze categorical data and was further analyzed using Two-tailed student $t$ test. Continuous variables with non-normal distribution was analyzed using the Mann-Whitney $U$ test. Statistical significance was set to $p$-value $<0.05$. The odds ratio (OR) with $95 \%$ confidence interval $(\mathrm{Cl})$ was calculated for comparing perioperative outcomes. Post hoc power analysis with a two-tailed alpha of 0.05 was performed between obese and non-obese groups to evaluate the power of detecting differences between patients experiencing any perioperative. A multivariable logistic regression model was used to determine the effects of sarcopenia, obesity, age, and gender on the likelihood of the occurrence of any AE.

\section{Results}

In total, there were 166 patients that met the inclusion criteria and 52 were removed based on the exclusion criteria. Final analysis included 114 patients (mean age $60.1 \pm 15.4$ years, 45 males, 69 females). The overall mean PMI was $495.0 \pm 182.9 \mathrm{~mm}^{2} / \mathrm{m}^{2}$. Interobserver and intraobserver reliabilities were considered excellent with ICC of 0.908 (95\% Cl 0.862-0.944) and 0.962 (95\% Cl 0.928-0.975), respectively. 54 patients were in the obese cohort and 60 patients in the non-obese cohort. The obese patients had a higher BMI and PMI compared to non-obese patients; otherwise, there were no significant differences in baseline demographics, comorbidities, presence of motor/sensory deficits, ambulatory status, indication for reoperation, or SSII between the two groups (Table 1). No significant difference between the perioperative outcomes was found among the obese and non-obese patients (Table 2). 
Table 1

Demographics and Characteristics of the Study Population

\begin{tabular}{|c|c|c|c|}
\hline Variable & Obese $(n=54)$ & Non-obese $(n=60)$ & $\begin{array}{l}P \text { - } \\
\text { value }\end{array}$ \\
\hline $\mathrm{BMI}\left(\mathrm{kg} / \mathrm{m}^{2}\right)$, mean $\pm \mathrm{SD}($ range $)$ & $36.2 \pm 5.4(30.0-51.7)$ & $25.8 \pm 3.0(16.0-29.8)$ & $<.001 *$ \\
\hline Male, n (\%) & $25(46.3)$ & $20(33.3)$ & 0.159 \\
\hline Age $(\mathrm{y})$, mean \pm SD (range) & $61.4 \pm 12.0(20-77)$ & $62.1 \pm 13.3(25-80)$ & 0.768 \\
\hline ASA Class, mean \pm SD (range) & $2.78 \pm 0.54(2-4)$ & $2.57 \pm 0.65(1-4)$ & 0.060 \\
\hline $\mathrm{PMI}\left(\mathrm{mm}^{2} / \mathrm{m}^{2}\right)$, mean $\pm \mathrm{SD}$ (range) & $\begin{array}{l}545.3 \pm 174.4(190.0- \\
941.7)\end{array}$ & $\begin{array}{l}449.6 \pm 179.8(152.2- \\
940.4)\end{array}$ & $0.004^{*}$ \\
\hline \multicolumn{4}{|l|}{ Comorbidities, n (\%) } \\
\hline DM & $11(20.4)$ & $10(16.7)$ & 0.611 \\
\hline Smoking & $7(13.0)$ & $7(11.7)$ & 0.834 \\
\hline $\begin{array}{l}\text { Cardiac (CHF, CAD, Atrial } \\
\text { fibrillation) }\end{array}$ & $11(20.4)$ & $14(23.3)$ & 0.704 \\
\hline CKD & $7(13.0)$ & $9(15.0)$ & 0.757 \\
\hline COPD & 19 (35.2) & $15(25.0)$ & 0.234 \\
\hline CVA & $1(1.9)$ & $1(1.7)$ & 0.936 \\
\hline PVD & $2(3.7)$ & $1(1.7)$ & 0.497 \\
\hline Cancer & $4(7.4)$ & $2(3.3)$ & 0.332 \\
\hline $\begin{array}{l}\text { No pre-operative motor deficits, } n \\
(\%)\end{array}$ & $41(75.9)$ & $39(65.0)$ & 0.204 \\
\hline $\begin{array}{l}\text { No pre-operative sensory deficits, } n \\
(\%)\end{array}$ & $35(64.8)$ & $30(50.0)$ & 0.110 \\
\hline \multicolumn{4}{|l|}{ Ambulatory status, n (\%) } \\
\hline $\begin{array}{l}\text { Ambulate without assistive } \\
\text { device }\end{array}$ & $16(29.6)$ & $20(33.3)$ & 0.674 \\
\hline Ambulate with assistive device & $30(55.6)$ & $23(38.3)$ & 0.066 \\
\hline Wheelchair bound & $8(14.8)$ & $7(11.7)$ & 0.617 \\
\hline Not recorded & $4(7.4)$ & $6(10.0)$ & 0.624 \\
\hline \multicolumn{4}{|l|}{ Indication for reoperation } \\
\hline Spinal stenosis & $22(40.7)$ & $29(48.3)$ & 0.418 \\
\hline
\end{tabular}




\begin{tabular}{|c|c|c|c|}
\hline Variable & Obese $(n=54)$ & Non-obese $(n=60)$ & $\begin{array}{l}P- \\
\text { value }\end{array}$ \\
\hline Disc disease & $13(24.1)$ & $16(26.7)$ & 0.749 \\
\hline Spondylolisthesis & $14(25.9)$ & $14(23.3)$ & 0.749 \\
\hline Trauma & $3(5.6)$ & $2(3.3)$ & 0.562 \\
\hline Deformity & $25(46.3)$ & $27(45.0)$ & 0.889 \\
\hline Adjacent segment disease & $22(40.7)$ & $24(40.0)$ & 0.936 \\
\hline Pseudarthrosis & $27(50.0)$ & $30(50.0)$ & 1.000 \\
\hline HNP & $3(5.6)$ & $3(5.0)$ & 0.897 \\
\hline Infection & $2(3.7)$ & $1(1.7)$ & 0.497 \\
\hline $\begin{array}{l}\text { SSIl of primary surgery, mean } \pm \text { SD } \\
\text { (range) }\end{array}$ & $11.9 \pm 6.3(3-32)$ & $13.9 \pm 5.9(3-32)$ & 0.067 \\
\hline $\begin{array}{l}\text { SSIl of revision surgery, mean } \pm \text { SD } \\
\text { (range) }\end{array}$ & $25.6 \pm 13.7(11-48)$ & $25.8 \pm 11.3(11-48)$ & 0.943 \\
\hline \multicolumn{4}{|l|}{ *Statistically significant values } \\
\hline \multicolumn{4}{|c|}{$\begin{array}{l}\text { PMI, psoas muscle index; ASA, American Society of Anesthesiologists; BMI, body mass index; DM, } \\
\text { diabetes mellitus; CHF, congestive heart failure; CAD, coronary artery disease; CKD, chronic kidney } \\
\text { disease; COPD, chronic obstructive pulmonary disease; CVA, cerebrovascular accident; PVD, peripheral } \\
\text { vascular disease; HNP, herniated nucleus pulposus; SSII, spine surgical invasiveness index }{ }^{18}\end{array}$} \\
\hline
\end{tabular}


Table 2

Comparison of Perioperative Outcomes

\begin{tabular}{|c|c|c|c|c|}
\hline Variable & Obese $(\mathrm{N}=54)$ & $\begin{array}{l}\text { Non-obese }(n= \\
60)\end{array}$ & $\begin{array}{l}P \\
\text { Value }\end{array}$ & $\begin{array}{l}\text { OR }(95 \% \\
\mathrm{Cl})\end{array}$ \\
\hline $\begin{array}{l}\text { Operative time (minutes), mean } \pm \text { SD } \\
\text { (range) }\end{array}$ & $\begin{array}{l}387.1 \pm 224.3 \\
(108-1160)\end{array}$ & $\begin{array}{l}372.2 \pm 172.7 \\
(122-937)\end{array}$ & 0.693 & N/A \\
\hline EBL $(m L)$, mean \pm SD (range) & $\begin{array}{l}1235.5 \pm 1792.4 \\
(75-12321)\end{array}$ & $\begin{array}{l}856.2 \pm 744.0 \\
(50-4600)\end{array}$ & 0.152 & N/A \\
\hline EBL > 1 L, n (\%) & $22(40.7)$ & $19(31.7)$ & 0.490 & $\begin{array}{l}1.29 \\
(0.63- \\
2.63)\end{array}$ \\
\hline $\begin{array}{l}\text { Intraoperative } \mathrm{pRBC} \text { transfusion units, } \\
\text { mean } \pm \mathrm{SD} \text { (range) }\end{array}$ & $2.2 \pm 2.6(0-15)$ & $1.7 \pm 1.6(0-8)$ & 0.238 & N/A \\
\hline $\begin{array}{l}\text { Post-operative LOS (days), mean } \pm \text { SD } \\
\text { (range) }\end{array}$ & $7.1 \pm 5.6(2-36)$ & $\begin{array}{l}6.3 \pm 4.0(1- \\
19)\end{array}$ & 0.375 & N/A \\
\hline \multicolumn{5}{|l|}{ Discharge Disposition, n (\%) } \\
\hline Home & $20(37.0)$ & 19 (31.7) & 0.547 & $\begin{array}{l}1.27 \\
(0.59- \\
2.76)\end{array}$ \\
\hline Facility & $34(63.0)$ & $41(68.3)$ & 0.547 & $\begin{array}{l}0.79 \\
(0.36- \\
1.71)\end{array}$ \\
\hline 30-day Reoperation, n (\%) & $4(7.4)$ & $5(8.3)$ & 0.855 & $\begin{array}{l}0.88 \\
(0.22- \\
3.46)\end{array}$ \\
\hline 30-day Readmission, n (\%) & $4(7.4)$ & $6(10.0)$ & 0.626 & $\begin{array}{l}0.72 \\
(0.19- \\
2.70)\end{array}$ \\
\hline In-hospital mortality, n (\%) & $0(0.0)$ & $1(1.7)$ & 0.539 & $\begin{array}{l}0.36 \\
(0.01- \\
9.12)\end{array}$ \\
\hline \multicolumn{5}{|l|}{ Perioperative Adverse Events, n (\%) } \\
\hline Any adverse events & $22(40.7)$ & $33(55.0)$ & 0.130 & $\begin{array}{l}0.56 \\
(0.27- \\
1.18)\end{array}$ \\
\hline $\begin{array}{l}\text { Post-op anemia requiring } \\
\text { transfusion }\end{array}$ & $8(14.8)$ & $18(30.0)$ & 0.058 & $\begin{array}{l}0.41 \\
(0.16- \\
1.03)\end{array}$ \\
\hline Cardiac (cardiac arrest, MI) & $1(1.9)$ & $2(3.3)$ & 0.487 & $\begin{array}{l}0.55 \\
(0.05- \\
6.21)\end{array}$ \\
\hline
\end{tabular}




\begin{tabular}{|c|c|c|c|c|}
\hline Variable & Obese $(N=54)$ & $\begin{array}{l}\text { Non-obese }(n= \\
60)\end{array}$ & $\begin{array}{l}P \\
\text { Value }\end{array}$ & $\begin{array}{l}\text { OR }(95 \% \\
\mathrm{Cl})\end{array}$ \\
\hline Sepsis & $0(0.0)$ & $1(1.7)$ & 0.539 & $\begin{array}{l}0.36 \\
(0.01- \\
9.12)\end{array}$ \\
\hline $\begin{array}{l}\text { Wound complication (dehiscence, } \\
\text { infection) }\end{array}$ & $4(7.4)$ & $7(11.7)$ & 0.763 & $\begin{array}{l}0.61 \\
(0.17- \\
2.20)\end{array}$ \\
\hline Delirium & $2(3.7)$ & $8(13.3)$ & 0.089 & $\begin{array}{l}0.25 \\
(0.05- \\
1.23)\end{array}$ \\
\hline Intra-op dural tear & $1(1.9)$ & $3(5.0)$ & 0.387 & $\begin{array}{l}0.36 \\
(0.04- \\
3.55)\end{array}$ \\
\hline AKI & $5(9.3)$ & $7(11.7)$ & 0.676 & $\begin{array}{l}0.77 \\
(0.23- \\
2.59)\end{array}$ \\
\hline Pneumonia & $3(5.6)$ & $6(10.0)$ & 0.386 & $\begin{array}{l}0.53 \\
(0.13- \\
2.23)\end{array}$ \\
\hline UTI & $2(3.7)$ & $9(15.0)$ & 0.059 & $\begin{array}{l}0.21 \\
(0.04- \\
1.06)\end{array}$ \\
\hline Urinary retention & $4(7.4)$ & $3(5.0)$ & 0.595 & $\begin{array}{l}1.52 \\
(0.32- \\
7.12)\end{array}$ \\
\hline Epidural hematoma & $2(3.7)$ & $0(0.0)$ & 0.262 & $\begin{array}{l}5.76 \\
(0.27- \\
122.7)\end{array}$ \\
\hline DVT & $4(7.4)$ & $6(10.0)$ & 0.626 & $\begin{array}{l}0.72 \\
(0.19- \\
2.70)\end{array}$ \\
\hline Nerve injury or paralysis & $2(3.7)$ & $0(0.0)$ & 0.262 & $\begin{array}{l}5.76 \\
(0.27- \\
122.7)\end{array}$ \\
\hline \multicolumn{5}{|c|}{$\begin{array}{l}\text { OR, odds ratio; EBL, estimated blood loss; } S D \text {, standard deviation; Cl, confidence interval; pRBC, } \\
\text { packed red blood cells; } L O S \text {, length of stay; MI, myocardial infarction; AKI, acute kidney injury; UTI, } \\
\text { urinary tract infection; } D V T \text {, deep vein thrombosis }\end{array}$} \\
\hline
\end{tabular}

Using the prior defined definition of sarcopenia as PMI $<500 \mathrm{~mm}^{2} / \mathrm{m}^{2}$ for males and $<412 \mathrm{~mm} / \mathrm{m}^{2}$ for females, 15 patients were considered obese and sarcopenic and 34 patients were considered non-obese and sarcopenic. ${ }^{14}$ No significant difference in perioperative outcomes was found between these patients (Table 3). 39 patients were considered obese and non-sarcopenic and 26 patients were considered non- 
obese and non-sarcopenic. No significant difference in perioperative outcomes was found between these patients (Table 4). 
Table 3

Comparison of Perioperative Outcomes among Sarcopenic Patients

\begin{tabular}{|c|c|c|c|c|}
\hline Variable & $\begin{array}{l}\text { Obese + } \\
\text { Sarcopenic ( }= \\
\text { 15) }\end{array}$ & $\begin{array}{l}\text { Non-obese }+ \\
\text { Sarcopenic }(n=34)\end{array}$ & $\begin{array}{l}P \\
\text { Value }\end{array}$ & $\begin{array}{l}\text { OR }(95 \% \\
\mathrm{Cl})\end{array}$ \\
\hline $\begin{array}{l}\text { Operative time (minutes), mean } \pm \\
\text { SD (range) }\end{array}$ & $\begin{array}{l}387.9 \pm 209.4 \\
(186-993)\end{array}$ & $\begin{array}{l}408.9 \pm 165.4 \\
(198-937)\end{array}$ & 0.734 & $\mathrm{~N} / \mathrm{A}$ \\
\hline EBL $(m L)$, mean \pm SD (range) & $\begin{array}{l}1118.3 \pm 1071.8 \\
(150-3500)\end{array}$ & $\begin{array}{l}926.2 \pm 605.6(50- \\
2400)\end{array}$ & 0.524 & $\mathrm{~N} / \mathrm{A}$ \\
\hline $\mathrm{EBL}>1 \mathrm{~L}, \mathrm{n}(\%)$ & $7(46.7)$ & $14(41.2)$ & 0.822 & $\begin{array}{l}1.13 \\
(0.38- \\
3.38)\end{array}$ \\
\hline $\begin{array}{l}\text { Intraoperative pRBC transfusion } \\
\text { units, mean } \pm \text { SD (range) }\end{array}$ & $2.2 \pm 2.2(0-7)$ & $1.8 \pm 1.4(0-6)$ & 0.278 & N/A \\
\hline $\begin{array}{l}\text { Post-operative LOS (days), mean } \pm \\
\text { SD (range) }\end{array}$ & $8.1 \pm 3.8(3-14)$ & $7.2 \pm 4.4(2-19)$ & 0.460 & $\mathrm{~N} / \mathrm{A}$ \\
\hline \multicolumn{5}{|l|}{ Discharge Disposition, n (\%) } \\
\hline Home & $3(20.0)$ & $4(11.8)$ & 0.453 & $\begin{array}{l}1.88 \\
(0.36- \\
9.66)\end{array}$ \\
\hline Facility & $12(80.0)$ & $30(88.2)$ & 0.453 & $\begin{array}{l}0.53 \\
(0.10- \\
2.75)\end{array}$ \\
\hline 30-day Reoperation, n (\%) & $3(20.0)$ & $4(11.8)$ & 0.453 & $\begin{array}{l}1.88 \\
(0.36- \\
9.66)\end{array}$ \\
\hline 30-day Readmission, n (\%) & $3(20.0)$ & $5(14.7)$ & 0.645 & $\begin{array}{l}1.45 \\
(0.30- \\
7.05)\end{array}$ \\
\hline In-hospital mortality, n (\%) & $0(0.0)$ & $1(2.9)$ & 0.844 & $\begin{array}{l}0.72 \\
(0.03- \\
18.7)\end{array}$ \\
\hline \multicolumn{5}{|l|}{ Perioperative Adverse Events, n (\%) } \\
\hline Any adverse events & $10(66.7)$ & $26(76.5)$ & 0.476 & $\begin{array}{l}0.62 \\
(0.16- \\
2.34)\end{array}$ \\
\hline $\begin{array}{l}\text { Post-op anemia requiring } \\
\text { transfusion }\end{array}$ & $5(33.3)$ & $15(44.1)$ & 0.481 & $\begin{array}{l}0.63 \\
(0.18- \\
2.25)\end{array}$ \\
\hline Cardiac (cardiac arrest, MI) & $1(6.7)$ & $1(2.9)$ & 0.554 & $\begin{array}{l}2.36 \\
(0.14- \\
40.4)\end{array}$ \\
\hline
\end{tabular}




\begin{tabular}{|c|c|c|c|c|}
\hline Variable & $\begin{array}{l}\text { Obese + } \\
\text { Sarcopenic (N = } \\
\text { 15) }\end{array}$ & $\begin{array}{l}\text { Non-obese }+ \\
\text { Sarcopenic }(n=34)\end{array}$ & $\begin{array}{l}P \\
\text { Value }\end{array}$ & $\begin{array}{l}\mathrm{OR}(95 \% \\
\mathrm{Cl})\end{array}$ \\
\hline Sepsis & $0(0.0)$ & $1(2.9)$ & 0.844 & $\begin{array}{l}0.72 \\
(0.03- \\
18.7)\end{array}$ \\
\hline $\begin{array}{l}\text { Wound complication } \\
\text { (dehiscence, infection) }\end{array}$ & $3(20.0)$ & $6(17.6)$ & 0.845 & $\begin{array}{l}1.17 \\
(0.25- \\
5.45)\end{array}$ \\
\hline Delirium & $2(13.3)$ & $7(20.6)$ & 0.549 & $\begin{array}{l}0.59 \\
(0.11- \\
3.26)\end{array}$ \\
\hline Intra-op dural tear & $1(6.7)$ & $2(5.9)$ & 0.916 & $\begin{array}{l}1.14 \\
(0.10- \\
13.7)\end{array}$ \\
\hline AKI & $3(20.0)$ & $9(26.5)$ & 0.628 & $\begin{array}{l}0.69 \\
(0.16- \\
3.04)\end{array}$ \\
\hline Pneumonia & $1(6.7)$ & $6(17.6)$ & 0.330 & $\begin{array}{l}0.33 \\
(0.04- \\
3.05)\end{array}$ \\
\hline UTI & $2(13.3)$ & $7(20.6)$ & 0.549 & $\begin{array}{l}0.59 \\
(0.11- \\
3.26)\end{array}$ \\
\hline Urinary retention & $2(13.3)$ & $3(8.8)$ & 0.633 & $\begin{array}{l}1.59 \\
(0.24- \\
10.7)\end{array}$ \\
\hline Epidural hematoma & $1(6.7)$ & $0(0.0)$ & 0.237 & $\begin{array}{l}7.14 \\
(0.27- \\
185.7)\end{array}$ \\
\hline DVT & $3(20.0)$ & $5(14.7)$ & 0.645 & $\begin{array}{l}1.45 \\
(0.30- \\
7.05)\end{array}$ \\
\hline Nerve injury or paralysis & $1(6.7)$ & $0(0.0)$ & 0.237 & $\begin{array}{l}7.14 \\
(0.27- \\
185.7)\end{array}$ \\
\hline \multicolumn{5}{|c|}{$\begin{array}{l}\text { OR, odds ratio; EBL, estimated blood loss; SD, standard deviation; CI, confidence interval; LOS, length } \\
\text { of stay; MI, myocardial infarction; AKI, acute kidney injury; UTI, urinary tract infection; DVT, deep vein } \\
\text { thrombosis }\end{array}$} \\
\hline
\end{tabular}


Table 4

Comparison of Perioperative Outcomes Among Non-Sarcopenic Patients

\begin{tabular}{|c|c|c|c|c|}
\hline Variable & $\begin{array}{l}\text { Obese }+ \text { Non- } \\
\text { Sarcopenic }(\mathrm{N}= \\
39)\end{array}$ & $\begin{array}{l}\text { Non-Obese + Non- } \\
\text { Sarcopenic }(N=26)\end{array}$ & $\stackrel{P}{\text { Value }}$ & $\begin{array}{l}\text { OR } \\
(95 \% \\
\text { Cl) }\end{array}$ \\
\hline $\begin{array}{l}\text { Operative time (minutes), mean } \pm \\
\text { SD (range) }\end{array}$ & $\begin{array}{l}386.8 \pm 232.4 \\
(108-1160)\end{array}$ & $\begin{array}{l}324.1 \pm 173.3(122- \\
847)\end{array}$ & 0.218 & N/A \\
\hline $\mathrm{EBL}(\mathrm{mL})$, mean $\pm \mathrm{SD}$ (range) & $\begin{array}{l}1280.6 \pm 2012.5 \\
(75-12321)\end{array}$ & $\begin{array}{l}764.7 \pm 898.3(50- \\
4600)\end{array}$ & 0.166 & N/A \\
\hline $\mathrm{EBL}>1 \mathrm{~L}, \mathrm{n}(\%)$ & $15(38.5)$ & $5(19.2)$ & 0.228 & $\begin{array}{l}2.00 \\
(0.65- \\
6.17)\end{array}$ \\
\hline $\begin{array}{l}\text { Intraoperative pRBC transfusion } \\
\text { units, mean } \pm \text { SD (range) }\end{array}$ & $2.2 \pm 2.8(0-15)$ & $1.6 \pm 1.8(0-8)$ & 0.135 & N/A \\
\hline $\begin{array}{l}\text { Post-operative LOS (days), mean } \pm \\
\text { SD (range) }\end{array}$ & $6.8 \pm 6.1(2-36)$ & $5.2 \pm 3.1(1-13)$ & 0.176 & N/A \\
\hline \multicolumn{5}{|l|}{ Discharge Disposition, n (\%) } \\
\hline Home & $17(43.6)$ & $15(57.7)$ & 0.267 & $\begin{array}{l}0.57 \\
(0.21- \\
1.55)\end{array}$ \\
\hline Facility & $22(56.4)$ & $11(42.3)$ & 0.267 & $\begin{array}{l}1.76 \\
(0.65- \\
4.81)\end{array}$ \\
\hline 30-day Reoperation, n (\%) & $1(2.6)$ & $1(3.8)$ & 0.771 & $\begin{array}{l}0.66 \\
(0.04- \\
11.0)\end{array}$ \\
\hline 30-day Readmission, n (\%) & $1(2.6)$ & $1(3.8)$ & 0.771 & $\begin{array}{l}0.66 \\
(0.04- \\
11.0)\end{array}$ \\
\hline In-hospital mortality, n (\%) & $0(0.0)$ & $0(0.0)$ & 0.843 & $\begin{array}{l}0.67 \\
(0.01- \\
34.9)\end{array}$ \\
\hline \multicolumn{5}{|l|}{$\begin{array}{l}\text { Perioperative Adverse Events, } \mathrm{n} \\
(\%)\end{array}$} \\
\hline Any adverse events & $12(30.8)$ & $7(26.9)$ & 0.739 & $\begin{array}{l}1.21 \\
(0.40- \\
3.63)\end{array}$ \\
\hline $\begin{array}{l}\text { Post-op anemia requiring } \\
\text { transfusion }\end{array}$ & $3(7.7)$ & $3(11.5)$ & 0.602 & $\begin{array}{l}0.64 \\
(0.12- \\
3.44)\end{array}$ \\
\hline Cardiac (cardiac arrest, MI) & $0(0.0)$ & $1(3.8)$ & 0.353 & $\begin{array}{l}0.22 \\
(0.01- \\
5.49)\end{array}$ \\
\hline
\end{tabular}




\begin{tabular}{|c|c|c|c|c|}
\hline Variable & $\begin{array}{l}\text { Obese }+ \text { Non- } \\
\text { Sarcopenic }(\mathrm{N}= \\
39)\end{array}$ & $\begin{array}{l}\text { Non-Obese + Non- } \\
\text { Sarcopenic }(N=26)\end{array}$ & $\begin{array}{l}P \\
\text { Value }\end{array}$ & $\begin{array}{l}\text { OR } \\
\text { (95\% } \\
\text { Cl) }\end{array}$ \\
\hline Sepsis & $0(0.0)$ & $0(0.0)$ & 0.843 & $\begin{array}{l}0.67 \\
(0.01- \\
34.9)\end{array}$ \\
\hline $\begin{array}{l}\text { Wound complication } \\
\text { (dehiscence, infection) }\end{array}$ & $1(2.6)$ & $1(3.8)$ & 0.771 & $\begin{array}{l}0.66 \\
(0.04- \\
11.0)\end{array}$ \\
\hline Delirium & $0(0.0)$ & $1(3.8)$ & 0.353 & $\begin{array}{l}0.22 \\
(0.01- \\
5.49)\end{array}$ \\
\hline Intra-op dural tear & $0(0.0)$ & $1(3.8)$ & 0.353 & $\begin{array}{l}0.22 \\
(0.01- \\
5.49)\end{array}$ \\
\hline AKI & $4(10.3)$ & $0(0.0)$ & 0.208 & $\begin{array}{l}6.72 \\
(0.35- \\
130.3)\end{array}$ \\
\hline Pneumonia & $2(5.1)$ & $0(0.0)$ & 0.421 & $\begin{array}{l}3.53 \\
(0.16- \\
76.6)\end{array}$ \\
\hline UTI & $0(0.0)$ & $0(0.0)$ & 0.843 & $\begin{array}{l}0.67 \\
(0.01- \\
34.9)\end{array}$ \\
\hline Urinary retention & $2(5.1)$ & $0(0.0)$ & 0.421 & $\begin{array}{l}3.53 \\
(0.16- \\
76.6)\end{array}$ \\
\hline Epidural hematoma & $1(2.6)$ & $0(0.0)$ & 0.661 & $\begin{array}{l}2.06 \\
(0.08- \\
52.7)\end{array}$ \\
\hline DVT & $1(2.6)$ & $1(3.8)$ & 0.771 & $\begin{array}{l}0.66 \\
(0.04- \\
11.0)\end{array}$ \\
\hline Nerve injury or paralysis & $1(2.6)$ & $0(0.0)$ & 0.661 & $\begin{array}{l}2.06 \\
(0.08- \\
52.7)\end{array}$ \\
\hline \multicolumn{5}{|c|}{$\begin{array}{l}\text { OR, odds ratio; EBL, estimated blood loss; SD, standard deviation; Cl, confidence interval; LOS, length } \\
\text { of stay; MI, myocardial infarction; AKI, acute kidney injury; UTI, urinary tract infection; DVT, deep vein } \\
\text { thrombosis }\end{array}$} \\
\hline
\end{tabular}

The multivariable logistic regression model was statistically significant, $\chi^{2}(4)=28.572, p<.0005 .72 .8 \%$ of the cases were correctly classified by the model, which also explained $29.6 \%$ (Nagelkerke $\mathrm{R}^{2}$ ) of the variance in any $A E$. The model revealed that sarcopenic individuals had a significantly higher likelihood for an AE than non-sarcopenic individuals (OR: 7.53, 95\% Cl: 3.05-18.60). Obesity, age, and gender did 
not have a significant effect in predicting AEs (Table 5). The post hoc power analysis between the obese and non-obese groups calculated a $95.2 \%$ power of detecting differences between patients experiencing any perioperative $\mathrm{AEs}$.

\section{Table 5}

Multivariable Logistic Regression Model to Assess Effects on Adverse Events

\begin{tabular}{|lllll|}
\hline Variable & $\boldsymbol{\beta}$ & SE & $\boldsymbol{P}$ Value & Adjusted OR (95\% Cl) \\
\hline Sarcopenia & 2.019 & 0.461 & $<0.001 *$ & $7.53(3.05-18.6)$ \\
\hline Obesity & 0.040 & 0.452 & 0.929 & $1.04(0.43-2.52)$ \\
\hline Age & 0.033 & 0.018 & 0.065 & $1.03(1.00-1.07)$ \\
Gender & -0.066 & 0.444 & 0.882 & $0.94(0.39-2.24)$ \\
*Statistically significant values & & \\
SE, standard error; OR, odds ratio; Cl, confidence interval
\end{tabular}

\section{Discussion}

In this study, the use of BMI as a predictor of perioperative AEs among patients undergoing complex thoracolumbar spine surgery was investigated. The multivariable logistic regression model confirmed that sarcopenic individuals were at a significantly higher likelihood to experience AE compared to nonsarcopenic individuals; however, there were no associations between BMI and perioperative AEs, 30-day readmission rates, 30-day re-operation rates, rate of discharge to a facility, or post-operative LOS among patients undergoing complex revision thoracolumbar spine surgery.

The relationship between obesity and perioperative AEs has been an area of debate over the past decade. Various studies have shown a significantly higher risk of AEs among obese patients after thoracolumbar surgery. ${ }^{17-21}$ The most recent study by Passias et al. determined that obese patients with a prior bariatric surgery had a significantly lower complication rate after thoracolumbar spine surgery compared with obese patients that did not undergo bariatric surgery. ${ }^{21}$ Our findings contradict these series of studies as our analysis demonstrated no significant difference in post-operative AEs between obese and non-obese patients. There are likely multiple reasons that our study had different results. First, our study performed a multivariable analysis with sarcopenia, measured by PMI, which demonstrated a significantly higher association with post-operative AEs. None of the prior studies that investigated the association between obesity and AEs included sarcopenia as a potential confounding variable. Second, our patient cohort consisted solely of complex revision cases, which is a particularly unique population with high complication rates at baseline. From our study analysis, it may be speculated that amongst these complex patients, pre-operative sarcopenia, and the associated debility and frailty, is a much better predictor for post-operative complications compared with the risks associated with obesity. Furthermore, studies have shown that although bariatric surgery is effective at lowering the BMI, this procedure may 
also induce sarcopenia post-operatively. ${ }^{22,23}$ Thus, with the known risks of sarcopenia shown within our study, it may be inadvisable to recommend bariatric surgery to improve BMI prior to undergoing complex revision thoracolumbar spine surgery.

A particularly important process among patients undergoing complex revision surgeries known to have high perioperative complications is preoperative risk stratification. Our previous study by Hirase et al. identified sarcopenia measured by $\mathrm{PMI}$ as a predictor of perioperative AEs among patients undergoing complex revision thoracolumbar spine surgery. ${ }^{14}$ Our multivariable analysis within this study confirmed these findings and also demonstrated that obesity is not associated with perioperative AEs within this patient population. This combination of findings will specifically assist spine surgeons during preoperative counseling and evaluation on two fronts. First, pre-operative optimization and overall conditioning to increase PMI among sarcopenic patients may be beneficial to preventing post-operative AE. Second, obese patients may not benefit from aggressive weight loss prior to these surgeries, particularly, as studies have shown that improper weight loss methods may lead to sarcopenia. ${ }^{24,25}$

Our study has several limitations. Data accuracy is contingent on charting accuracy due to the retrospective nature of the study and may be susceptible to certain selection bias. Our results obtained from a single-center data may not be completely reflective of outcomes from other institutions due to variations in surgical technique or management. Our study also consisted of a relatively small patient cohort that may have led to underpowering to detect certain associations; however, our post hoc power analysis demonstrated that the absence of observed difference is unlikely due to lack of power. Furthermore, there may have been small discrepancies in inter-scan agreement between CT and MRI scans used to obtain the PMI; however, this is a previously established method with studies showing good inter-scan reliability with an ICC of $0.821 .^{14,26}$ Thus, to increase the power of the study, patients were included if they received either a CT or an MRI pre-operatively. Furthermore, although this study demonstrates that obesity is not a predictor of post-operative AEs within this surgical population, we cannot directly conclude that weight loss will not be beneficial in preventing post-operative AEs.

Therefore, further studies that investigate the external validity of this study may be beneficial prior to application in practice.

In spite of the aformentioned limitations, to the best of our knowledge, this is the largest study examining the relationship between obesity and perioperative outcomes among patients receiving complex revision thoracolumbar spine surgery. This study will serve to assist spine surgeons with additional information during pre-operative counseling and evaluation regarding risks and benefits associated with sarcopenia and obesity. For patients undergoing complex revision thoracolumbar spine surgery, our findings may suggest that the benefits of weight loss among obese patients may not outweigh the risk of inducing sarcopenia prior to the operation.

\section{Conclusions}


Obesity is not associated with perioperative AEs, 30-day readmission rates, 30-day re-operation rates, rate of discharge to a facility, or post-operative length of stay (LOS) among patients undergoing complex revision thoracolumbar spine surgery.

\section{Abbreviations}

AEs

adverse events; $\mathrm{PMI}=$ psoas muscle index; $\mathrm{LOS}=$ length of stay; $\mathrm{BMI}=$ body mass index; $\mathrm{SSII}=$ spine surgical invasiveness index; ASA = American Anesthesiologists' Society; EMR = electronic medical records; $\mathrm{EBL}=$ estimated blood loss; $\mathrm{CSA}=$ cross-sectional area; $\mathrm{ICC}=$ intraclass correlation coefficient; $\mathrm{AKI}=$ acute kidney injury; $\mathrm{UTI}=$ urinary tract infection; $\mathrm{DVT}=$ deep vein thrombosis; $\mathrm{OR}=$ odds ratio; $\mathrm{Cl}=$ confidence interval

\section{Declarations}

Ethics approval and consent to participate: The study protocol was approved through the Houston Methodist Hospital institutional review board on April 13, 2020 (PRO00024733) with a waiver for informed consent attributed to the study's retrospective observational nature.

Consent for publication: Written informed consent for publication of their clinical details and/or clinical images was obtained from the patient/parent/guardian/ relative of the patient. A copy of the consent form is available for review by the Editor of this journal.

Availability of data and material: Available upon request to corresponding author

\section{Competing interests:}

Takashi Hirase, Jeremiah Ling, Varan Haghshenas, Richard Fuld III, David Dong, and B Christoph Meyer declare no potential conflicts of interest with respect to research, authorship, and/or publication of this article.

Rex AW Marco has the following disclosures:

DePuy, A Johnson \& Johnson Company: Paid presenter or speaker; Globus Medical: Paid presenter or speaker; Musculoskeletal Tumor Society: Board or committee member; Stryker: Paid presenter or speaker; Synaptive Medical: Paid presenter or speaker

Darrell S Hanson has the following disclosures:

DePuy, A Johnson \& Johnson Company: IP royalties; Paid consultant; Paid presenter or speaker; Medtronic Sofamor Danek: Paid consultant; Paid presenter or speaker

Funding: None 
Author's Contributions: All authors made substantial contributions to the conception and design of work and provided final approval of the version to be published. Specific additional individual contribution includes: Takashi Hirase: Interpretation and analysis of data for the work. Drafted the introduction, the overall work outline, and made critical final revisions. Jeremiah Ling: Lead the acquisition of data and drafted the results section. Varan Haghshenas: Drafted a portion of the discussion section and made critical final revisions. Richard Fuld III: Assisted in the acquisition of data and drafted the results section. David Dong: Assisted in the acquisition of data and drafted the results section. Darrell Hanson: Senior surgeon with substantial contribution to design of work and made critical final revisions and approval of the version to be published. B Christoph Meyer: Senior surgeon with substantial contribution to design of work and made critical final revisions and approval of the version to be published. Rex AW Marco: Senior surgeon with substantial contribution to design of work and made critical final revisions and approval of the version to be published

Acknowledgements: Takashi Hirase was supported by a Burroughs Wellcome Fund Physician Scientist Institutional Award to the Texas A\&M University Academy of Physician Scientists

\section{References}

1. Flegal KM, Carroll MD, Kuczmarski RJ, \& Johnson CL. Overweight and obesity in the United States: prevalence and trends, 1960-1994. Int J Obes Relat Metab Disord. 1998;22(1):39-47.

2. Clinical Guidelines on the Identification, Evaluation, and Treatment of Overweight and Obesity in Adults-The Evidence Report. National Institutes of Health. Obes Res. 1998;6 Suppl 2:51S-209S.

3. Purnell, J. Q. Definitions, Classification, and Epidemiology of Obesity. In: K. R. Feingold Anawalt B, Boyce A, Chrousos G, de Herder WW, Dhatariya K, Dungan K, Grossman A, Hershman JM, et. al. 2018. http://www.endotext.org. Accessed Oct 2020.

4. Park, P., Upadhyaya, C., Garton, H. J., \& Foley, K. T. The impact of minimally invasive spine surgery on perioperative complications in overweight or obese patients. Neurosurgery. 2008;62(3):693-699.

5. Cao, J., Kong, L., Meng, F., Zhang, Y., \& Shen, Y. Impact of obesity on lumbar spinal surgery outcomes. J Clin Neurosci. 2016;28:1-6.

6. Manson, N. A., Green, A. J., \& Abraham, E. P. Elevated Patient Body Mass Index Does Not Negatively Affect Self-Reported Outcomes of Thoracolumbar Surgery: Results of a Comparative Observational Study with Minimum 1-Year Follow-Up. Global Spine J. 2016;6(2):108-117.

7. Shamji, M. F., Parker, S., Cook, C., Pietrobon, R., Brown, C., \& Isaacs, R. E. Impact of body habitus on perioperative morbidity associated with fusion of the thoracolumbar and lumbar spine. Neurosurgery. 2009;65(3):490-498.

8. Yadla S, Malone J, Campbell PG, Maltenfort MG, Harrop JS, Sharan AD, et al. Obesity and spine surgery: reassessment based on a prospective evaluation of perioperative complications in elective degenerative thoracolumbar procedures. Spine J. 2010;10(7):581-587. 
9. Varshneya K, Pangal DJ, Stienen MN, Ho AL, Fatemi P, Medress ZA, et al. Postoperative Complication Burden, Revision Risk, and Health Care Use in Obese Patients Undergoing Primary Adult Thoracolumbar Deformity Surgery. Global Spine J. 2021;11(3):345-350.

10. Kim SS, Michelsen CB. Revision Surgery for failed back surgery syndrome. Spine (Phila Pa 1976). 1992;17(8):957-60.

11. Fritsch EW, Heisel J, Rupp S. The failed back surgery syndrome: reasons, intraoperative findings, and long-term results: a report of 182 operative treatments. Spine (Phila Pa 1976). 1996;21(5):626-33.

12. Sansur CA, Smith JS, Coe JD, Glassman SD, Berven SH, Polly DW Jr, et al. Scoliosis research society morbidity and mortality of adult scoliosis surgery. Spine (Phila Pa 1976). 2011;36(9):E593-7.

13. Camino Willhuber G, Elizondo C, Slullitel P. Analysis of Postoperative Complications in Spinal Surgery, Hospital Length of Stay, and Unplanned Readmission: Application of Dindo-Clavien Classification to Spine Surgery. Global Spine J. 2019;9(3):279-86.

14. Hirase T, Haghshenas V, Bratescu R, Dong D, Kuo PH, Rashid A, et al. Sarcopenia Predicts Perioperative Adverse Events Following Complex Revision Surgery for the Thoracolumbar Spine. Spine J. 2021 Jun;21(6):1001-1009.

15. Moskven E, Bourassa-Moreau E, Charest-Morin R, Flexman A, Street J. The impact of frailty and sarcopenia on postoperative outcomes in adult spine surgery. A systematic review of the literature. Spine J. 2018; 18(12):2354-69.

16. Koo TK, Li MY. A Guideline of Selecting and Reporting Intraclass Correlation Coefficients for Reliability Research. J Chiropr Med. 2016;15(2):155-63.

17. Knutsson BB, Michaëlsson K, Sandén B. Obesity is associated with inferior results after surgery for lumbar spinal stenosis: a study of 2633 patients from the Swedish spine register. Spine (Phila Pa 1976). 2013;38(5):435-441

18. Marquez-Lara A, Nandyala SV, Sankaranarayanan S, Noureldin M, Singh K. Body mass index as a predictor of complications and mortality after lumbar spine surgery. Spine (Phila Pa 1976). 2014;39(10):798-804

19. Buerba RA, Fu MC, Gruskay JA, Long WD 3rd, Grauer JN. Obese class III patients at significantly greater risk of multiple complications after lumbar surgery: an analysis of 10,387 patients in the ACS NSQIP database. Spine J. 2013;14(9):2008-2018

20. Christou NV, Sampalis JS, Liberman M, Look D, Auger S, McLean AP, MacLean LD. Surgery decreases long-term mortality, morbidity, and health care use in morbidly obese patients. Ann Surg. 2004;240(3):416-23-4

21. Passias PG, Horn SR, Vasquez-Montes D, Shepard N, Segreto FA, Bortz CA, et al. Prior bariatric surgery lowers complication rates following spine surgery in obese patients. Acta Neurochir (Wien). 2018;160(12):2459-2465.

22. Pekař $M$, Pekařová $A$, Bužga $M$, Holéczy $P$, Soltes $M$. The risk of sarcopenia 24 months after bariatric surgery - assessment by dual energy X-ray absorptiometry (DEXA): a prospective study. Wideochir Inne Tech Maloinwazyjne. 2020 Dec;15(4):583-587. 
23. Voican CS, Lebrun A, Maitre S, Lainas P, Lamouri K, Njike-Nakseu M, et al. Predictive score of sarcopenia occurrence one year after bariatric surgery in severely obese patients. PLoS One. 2018;13(5):e0197248.

24. Miller SL, Wolfe RR. The danger of weight loss in the elderly. J Nutr Health Aging. 2008;12(7):48791.

25. Tournadre A, Vial G, Capel F, Soubrier M, Boirie Y. Sarcopenia. Joint Bone Spine. 2019;86(3):309-314.

26. Park J, Gil JR, Shin Y, Won SE, Huh J, You MW, Park HJ, et al. Reliable and robust method for abdominal muscle mass quantification using CT/MRI: An explorative study in healthy subjects. PLoS One. 2019;14(9):e0222042. 